

PHYsICS Richard Feynman's lectures, still loved 50 years on $\mathbf{p . 3 0}$
EDUCATION Weighing the case for gene streaming in schools p.32
HEALTH Microbe-rich life with the Hadzabe huntergatherers of Tanzania p.33
OBITUARY Leonard Herzenberg, immunology technology pioneer, remembered p.34



The Moon has a similar composition to the outer portions of Earth.

\title{
Lunar conspiracies
}

\section{Current theories on the formation of the Moon owe too much to cosmic coincidences, says Robin Canup. She calls for better models and a mission to Venus.}

$\mathrm{T}$ he Moon is more than just a familiar sight in our skies. It dictates conditions on Earth. The Moon is large enough to stabilize our planet's rotation, holding Earth's polar axis steady to within a few degrees. Without it, the current Earth's tilt would vary chaotically by tens of degrees. Such large variations might not preclude life, but would lead to a vastly different climate.

Knowing how the Moon was made is central to understanding Earth and the formation of other planets. Since the 1980s, work on lunar origins has focused on the 'giant-impact' theory. This proposes that the collision of another planet-sized body with the forming Earth generated a disk of debris that coalesced into the Moon. Such giant collisions were common in the Solar System during the final stages of Earth's formation 4.5 billion years ago.

But we still do not understand in detail how an impact could have produced our Earth and Moon. In the past few years, computer simulations, isotope analyses of rocks and data from lunar missions have raised the possibility

\section{$\rightarrow$ NATURE.COM}

\section{For more on the}

Moon's origins, see:

go.nature.com/5foh6i of new mechanisms to explain the observed characteristics of the Earth-Moon system.

The main challenge is to simultaneously account for the pair's dynamics - in particular, the total angular momentum contained in the Moon's orbit and Earth's 24-hour day - while also reconciling their many compositional similarities and few key differences. The collision of a large impactor with Earth can supply the needed angular momentum, but it also creates a disk of material derived largely from the impactor. If the infalling body had a different composition from Earth, as seems probable given that most objects in 
HOW TO MAKE | Simulations reveal how a giant collision between two similarly sized planets ('half-Earths') AMOON might explain why the Moon has a similar composition to Earth's mantle. The violent crash
blended both planets to produce Earth and a disk of hot debris that coalesced to form the Moon (blue to red spans temperatures from below 2,000 kelvin to more than 6,400 kelvin) $\rightarrow$ FOR AN ANIMATED VERSION SEE: go.nature.com/hndjnq
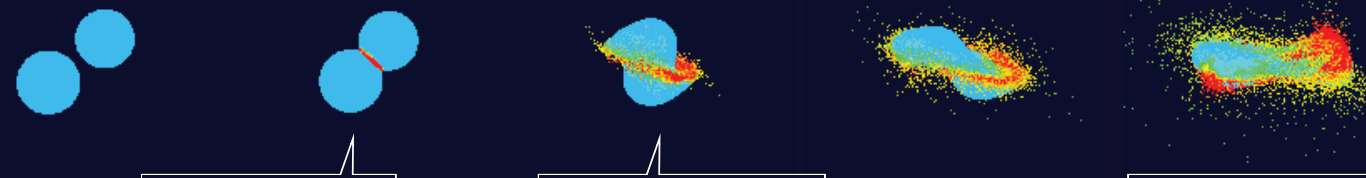

1

The bodies are sheared

by the impact.

the inner Solar System do, then why is the composition of the Moon so similar to the outer portions of our planet?

So far, the solutions proposed appeal to extra processes - such as extensive mixing of materials from both bodies or a later gravitational resonance with the Sun - the feasibility of which are unclear. Planetary scientists need to double modelling efforts of the Earth-Moon system and identify chemical signatures in lunar and terrestrial rocks that could rule out some scenarios or suggest alternatives.

\section{MORE ALIKE THAN DIFFERENT}

There are clear differences in the compositions of the Moon and Earth. Earth's core is rich in iron, which comprises about $30 \%$ of the planet's mass. By contrast, iron contributes less than $10 \%$ to the mass of the Moon. The Moon is also less rich in elements that vaporize readily, such as potassium, suggesting that they may have boiled off and been lost as the Moon formed from the hot disk.

Analyses of samples brought back by the Apollo missions in the 1970s have shown that the silicate mantles of the Moon and Earth share identical oxygen isotope compositions (to within measurement precision $)^{1}$, distinct from those of meteorites from Mars and from most of the asteroid belt. In recent years the similarities have mounted. The chromium, titanium, tungsten and silicon isotope compositions of the Moon and Earth now also seem to be indistinguishable ${ }^{2-4}$.

Gravity observations of the Moon from NASA's Gravity Recovery and Interior Laboratory (GRAIL) spacecraft, combined with topography data from NASA's Lunar Reconnaissance Orbiter, have reduced estimates for the thickness of the Moon's crust and its aluminium abundance. These measurements suggest that refractory elements (metals with high condensation temperatures) are similarly abundant in both bodies ${ }^{5}$, rather than more prevalent in the Moon, as previously thought.

Collectively, these data imply that either the Moon formed from material originating directly from Earth's mantle, or that the Moon and the silicate portion of Earth each formed from an identical mix of material. Special circumstances seem to be required in either case.

\section{IMPACT MODELS}

Moon-forming collisions are studied through simulations. Because the energy caused by the impact of the colliding planets is high enough to melt or even partially vaporize them, pressure forces and phase changes are incorporated into the models. Gravitational interactions and torques are also included because the collision distorts the planets and ejects debris into a disk. Mantle and core materials need to be tracked.

In the canonical giant-impact model, developed since the late 1970 s, the Moon is explained as the product of a slow, glancing blow from a Mars-sized body - about $10-15 \%$ of Earth's mass - on the early Earth ${ }^{6}$. The collision left Earth spinning rapidly, once every five hours, with the Moon orbiting close to Earth. Gravitational interactions and torques then caused the Moon's orbit to expand and Earth's rotation to slow to our current 24-hour day. This model is consistent with the Moon's mass, its lack of iron and the angular



The Moon's gravity field as mapped by NASA's Gravity Recovery and Interior Laboratory. momentum of the Earth-Moon system.

More detailed chemical properties are harder to explain. The giant-impact model has the Moon condensing from material in a disk, which in canonical impacts is derived primarily from the impactor's mantle. But it is improbable that the impactor had the same composition as the early Earth. The oxygen isotope composition of Mars, for example, differs from that of the Earth by more than a factor of 50 (ref. 1). If the impactor was as different from Earth as Mars is, its signature would still be detectable in the Moon, even after a giant collision.

An elegant solution, known as equilibration, was proposed in 2007 by planetary scientists Kaveh Pahlevan and David Stevenson ${ }^{7}$. They suggested that vapour from the disk and the outer Earth mixed after the impact but before the Moon formed. But there are difficulties with this proposal. It takes at least 100 years for vapour from the disk and Earth to diffuse and mix thoroughly. But in that time the distant portions of the disk should have begun to coalesce into the Moon ${ }^{8}$.

It is possible that the inner portions of the Moon could have retained the composition of the impactor. There are no signatures of this in lunar rocks; however, these represent only the outermost few hundred kilometres of the satellite's interior. Another conundrum is that volatile elements in the post-collision vapour would be expected to mix more readily than refractory ones, yet both oxygen and titanium, for instance, are identical in the two bodies.

In 2012, planetary scientists Matija Ćuk and Sarah Stewart ${ }^{9}$ broadened the range of possible Moonforming impacts. Earth's oblate shape causes the orientation of the Moon's elliptical orbit to gradually rotate with a period that lengthens as the Moon's orbit expands - a process known as precession. Cuk and Stewart showed that a resonant state between the Moon and the Sun that occurs when the lunar precession period matches the one-year period of Earth's orbit could - if it persists long enough — halve Earth's spin rate. Impacts of higher angular momentum then become viable, including 


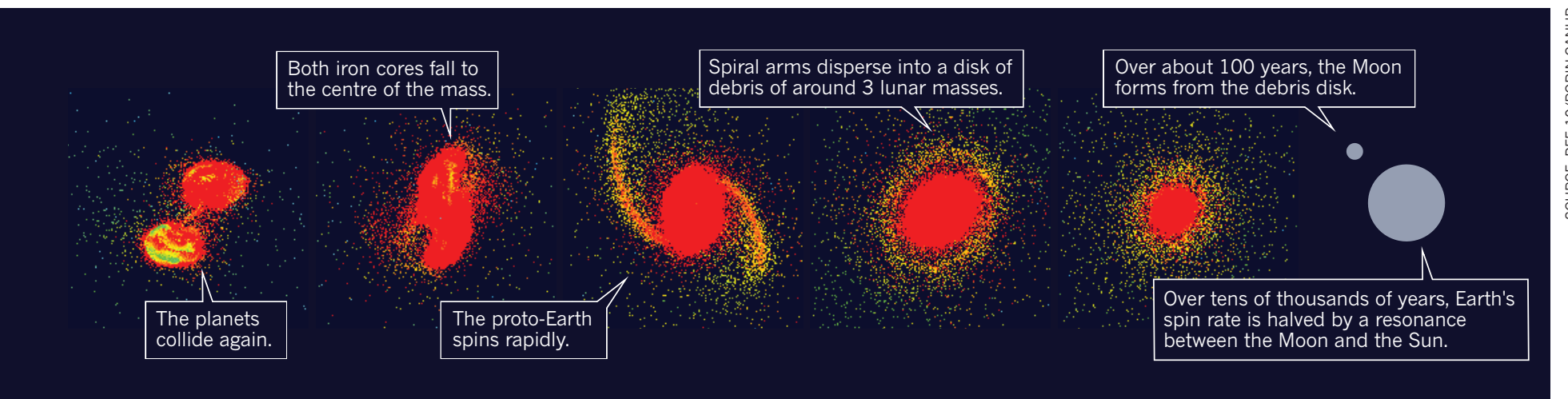

two cases that can produce a disk with the composition of Earth's mantle.

The 'fast-spinning Earth' scenario, proposed by Cuk and Stewart ${ }^{9}$, invokes the collision of an object slightly smaller than Mars with an Earth that is already rotating with a 2-2.5-hour day owing to a previous large impact. Because Earth is spinning close to the critical rate at which it becomes unstable, the Moon-forming impact ejects part of Earth's mantle into orbit, leading to a disk.

Also in 2012, I proposed the 'half-Earth impactor' scenario ${ }^{10}$. Here, the Moon arises from a collision between two planets, each of about half of Earth's mass (see 'How to make a Moon'). Both final planet and disk then comprise about half impactor and half target material. This model is simpler than the fast-spinning-Earth model because it does not require a specific prior large impact. But it demands a large impactor, and so may still be less probable than the canonical impact.

Both 2012 models account for the similar oxygen, chromium and titanium compositions of the Moon and Earth. To explain similarities in silicon and tungsten - elements that interact with metals - both models require that the impactor's iron core remains largely intact as it descends through Earth's mantle to merge with Earth's core, avoiding substantial metal-silicate interactions. But it remains unclear whether the resonance mechanism needed to slow Earth's rotation in these more extreme scenarios is likely or requires an improbably narrow range of conditions. In other words, is the origin of our Moon a rarer event than we believed, or are we missing something?

\section{FUTURE DIRECTIONS}

Lunar-origin studies are in flux. No current impact model stands out as more compelling than the rest. Progress in several areas is needed to rule out some theories, support others or direct us to new ones.

First, a better understanding of what happened between the formation of the disk and the accumulation of the Moon from the disk is essential, because this phase established the Moon's properties. Did mixing homogenize the composition of the disk and the planet before the Moon formed? Were volatile elements lost from the disk, and, if so, did the pattern of loss vary with the disk's temperature? Canonical impacts produce a mostly liquid disk whereas in the highangular-momentum impacts, the disks are initially largely vapour. Such disk-evolution models are technically challenging and will require a multidisciplinary approach incorporating both dynamics and chemistry.

Second, the likelihood that a resonance altered the Earth-Moon angular momentum needs to be assessed for a variety of physical states of the early Earth and Moon and using state-of-the-art models for the tidal interactions between them.

Finally, further isotopic comparisons of lunar and terrestrial materials would be extremely valuable. They should include highly refractory elements, such as calcium, to test the equilibration model. Finding that an element that could not have mixed in a vapour phase in 100 years is the same in the Moon and Earth but different in Mars would argue against equilibration; finding EarthMoon isotopic differences in such a highly refractory element would support it.

Oxygen provides arguably the most important isotopic constraint on lunar formation. The distinct oxygen isotopic compositions of the Earth-Moon system, Mars and most meteorites reflect different initial compositional reservoirs in the inner Solar System. This simplifies the interpretation of oxygen compositions compared with elements such as silicon, whose isotopic abundances are affected by later planet-forming processes (such as crustal extraction). Increasing the precision of oxygen isotope measurements could potentially rule out some impact scenarios.

It remains troubling that all of the current impact models invoke a process after the impact to effectively erase a primary outcome of the event - either by changing the disk's composition through mixing for the canonical impact, or by changing Earth's spin rate for the high-angular-momentum narratives.

Sequences of events do occur in nature, and yet we strive to avoid such complexity in our models. We seek the simplest possible

solution, as a matter of scientific aesthetics and because simple solutions are often more probable. As the number of steps increases, the likelihood of a particular sequence decreases. Current impact models are more complex and seem less probable than the original giant-impact concept.

A clue may lie in Venus. The assumption that the Moon-forming impactor had a composition very different from that of Earth is

"Is the origin of our Moon a rarer event than we believed, or are we missing something?"

largely based on what we know about Mars. We do not know the isotopic composition of Venus, the planet most similar to Earth in both mass and distance from the Sun. If Venus's composition proves similar to that of Earth and the Moon, Mars would then seem to be an outlier, and an impactor composition akin to Earth's would be more probable, removing many objections to the canonical impact.

Determining the isotopic composition of Venus's key elements will probably require a mission to the planet. Such a tantalizing prospect reminds us how much there is still to learn in our Solar System

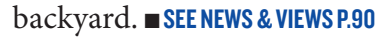

Robin Canup is associate vice-president of the Planetary Science Directorate of Southwest Research Institute, Boulder, Colorado.

e-mail:robin@boulder.swri.edu

1. Wiechert, U. et al. Science 294, 345-348 (2001)

2. Touboul, M., Kleine, T., Bourdon, B., Palme, H. \& Wieler, R. Nature 450, 1206-1209 (2007).

3. Armytage, R. M. G., Georg, R. B., Williams, H. M. \& Halliday, A. N. Geochim. Cosmochim. Acta 77 504-514 (2012).

4. Zhang, J., Dauphas, N., Davis, A. M., Leya, I. \& Fedkin, A. Nature Geosci. 5, 251-255 (2012)

5. Wieczorek, M. A. et al. Science 339, 671-675 (2013).

6. Canup, R. M. \& Asphaug, E. Nature 412, 708-712 (2001).

7. Pahlevan, K. \& Stevenson, D. J. Earth Planet. Sci. Lett. 262, 438-449 (2007).

8. Salmon, J. \& Canup, R. M. Astrophys. J. 760, 83 (2012).

9. Ćuk, M. \& Stewart, S. T. Science 338, 1047-1052 (2012).

10.Canup, R. M. Science 338, 1052-1055 (2012). 